\title{
Pediatric Cushing disease: disparities in disease severity and outcomes in the Hispanic and African-American populations
}

\author{
Alexandra Gkourogianni ${ }^{1}$, Ninet Sinaii ${ }^{2}$, Sharon H. Jackson ${ }^{3}$, Alexander S. Karageorgiadis ${ }^{1}$, Charalampos Lyssikatos ${ }^{1}$, \\ Elena Belyavskaya ${ }^{1}$, Margaret F. Keil ${ }^{1}$, Mihail Zilbermint ${ }^{1}$, Prashant Chittiboina ${ }^{4}$, Constantine A. Stratakis ${ }^{1}$ and \\ Maya B. Lodish ${ }^{1}$
}

BACKGROUND: Little is known about the contribution of racial and socioeconomic disparities to severity and outcomes in children with Cushing disease (CD).

METHODS: A total of 129 children with CD, 45 Hispanic/ Latino or African-American (HI/AA) and 84 non-Hispanic White (non-HW), were included in this study. A 10-point index for rating severity ( $C D$ severity) incorporated the degree of hypercortisolemia, glucose tolerance, hypertension, anthropomorphic measurements, disease duration, and tumor characteristics. Race, ethnicity, age, gender, local obesity prevalence, estimated median income, and access to care were assessed in regression analyses of CD severity.

RESULTS: The mean CD severity in the HI/AA group was worse than that in the non-HW group ( $4.9 \pm 2.0$ vs. $4.1 \pm 1.9$, $P=0.023)$; driving factors included higher cortisol levels and larger tumor size. Multiple regression models confirmed that race $(P=0.027)$ and older age $(P=0.014)$ were the most important predictors of worse CD severity. When followed up a median of 2.3 years after surgery, the relative risk for persistent CD combined with recurrence was 2.8 times higher in the HI/AA group compared with that in the non-HW group (95\% confidence interval: 1.2-6.5).

CONCLUSION: Our data show that the driving forces for the discrepancy in severity of CD are older age and race/ethnicity. Importantly, the risk for persistent and recurrent CD was higher in minority children.

A n estimated 1-1.5 per million children are affected by Cushing syndrome every year; of those cases, $75-80 \%$ are caused by an adrenocorticotropic hormone-secreting pituitary tumor (Cushing disease, CD) (1). Prolonged exposure to excess glucocorticoids leads to obesity, growth deceleration, striae, muscle weakness, hypertension, impaired glucose intolerance, osteopenia/osteoporosis, and alterations in cognitive function and mood (2). These tumors are typically benign, and early identification and surgical resection may lead to long-term remission and cure (3). Importantly, improved outcomes in children with $\mathrm{CD}$ are associated with younger age at surgery, smaller adenomas, and lack of dural invasion (3).

Racial, ethnic, and socioeconomic disparities have been investigated in a number of diseases associated with tumors; significant differences in disease incidence, prevalence, and mortality have been identified (4-6). Even as treatment and detection of tumors have improved, African-Americans continue to experience lower survival rates than Whites for all cancers (7). For the most common childhood cancer, acute lymphoblastic leukemia, Black, and Hispanic children have worse survival than do white children; in addition, socioeconomic status (SES) is associated with poor survival in childhood leukemia $(8,9)$. Five-year survival rates among Hispanic (HI; 74\%) and African-American (AA; 75\%) children remain poorer than those among non-Hispanic Whites (non-HW; 85\%) for CNS malignancies as well as for many other tumors $(5,10)$. Although disproportionate morbidity and mortality have been shown for more common cancers, no prior studies have examined differences in severity of presentation for children with $\mathrm{CD}$. In addition to looking at individual clinical parameters and comparing them between racial groups, we also introduced a combined pediatric $\mathrm{CD}$ severity score (CD severity) as a tool for research purposes. As has been done for other disease states when severity scores were being formulated, we looked at the co-morbidities associated with $\mathrm{CD}$. Each of the individual factors used in the scoring system has independently been associated with an increased risk for a poor outcome in patients (degree of hypercortisolemia, impaired glucose tolerance, hypertension, height impairment, body mass index (BMI), delay in diagnosis, and larger tumor) $(3,11,12)$. Our severity score aims to quantify the magnitude of glucocorticoid exposure to characterize the severity of illness. In addition, inhibition of linear growth by exogenous steroids has been shown to be dose-dependent, a unique factor in children when compared with adults with $\mathrm{CD}$. In adults with $\mathrm{CD}$, hypertension and

${ }^{1}$ Section on Endocrinology and Genetics, Developmental Endocrinology Branch, and Pediatric Endocrinology Inter-Institute Training Program, Eunice Kennedy Shriver National Institute of Child Health and Human Development, National Institutes of Health, Bethesda, Maryland; ${ }^{2}$ Biostatistics and Clinical Epidemiology Service, NIH Clinical Center, Bethesda, Maryland; ${ }^{3}$ National Institute on Minority and Health Disparities, NIH, Bethesda, Maryland; ${ }^{4}$ Surgical Neurology Branch, National Institute of Neurological Disorders and Stroke, NIH, Bethesda, Maryland. Correspondence: Maya B. Lodish (lodishma@mail.nih.gov)

Received 2 November 2016; accepted 12 February 2017; advance online publication 17 May 2017. doi:10.1038/pr.2017.58 
Table 1. Cushing disease severity score

\begin{tabular}{|c|c|c|c|}
\hline Severity score component & To capture & Cutoff & Component score \\
\hline S1 & Degree of hypercortisolemia & $\begin{array}{l}\text { MSC and/or } 24-h \text { UFC }<50 \% \text { ile } \\
\text { MSC and/or } 24-h \text { UFC } \geqslant 50-<75 \% \text { ile } \\
\text { MSC and/or } 24-h \text { UFC } \geqslant 75 \% \text { ile }\end{array}$ & $\begin{array}{l}0 \\
1 \\
2\end{array}$ \\
\hline S2 & IGT & $\begin{array}{l}\mathrm{FPG}<100 \mathrm{mg} / \mathrm{dl} \\
\mathrm{FPG} \geqslant 100 \mathrm{mg} / \mathrm{dl} \\
\mathrm{FPG} \geqslant 126 \mathrm{mg} / \mathrm{dl} \text { and/or DM-Dx }\end{array}$ & $\begin{array}{l}0 \\
1 \\
2\end{array}$ \\
\hline S3 & Hypertension & $\begin{array}{l}\text { SBP and DBP } z \text { score both }<2 \\
\text { SBP or DBP } z \text { score } \geqslant 2 \\
\text { HTN-Tx }\end{array}$ & $\begin{array}{l}0 \\
1 \\
2\end{array}$ \\
\hline S4 & Height z score & $\begin{array}{l}\text { Height } z \text { score }>-0.5 \\
\text { Height } z \text { score } \leqslant-0.5\end{array}$ & $\begin{array}{l}0 \\
1\end{array}$ \\
\hline S5 & BMI z score & $\begin{array}{l}\text { BMI } z \text { score }<2 \\
\text { BMI } z \text { score } \geqslant 2\end{array}$ & $\begin{array}{l}0 \\
1\end{array}$ \\
\hline S6 & $t$ (to diagnosis) & $\begin{array}{l}<3 \text { years } \\
\geqslant 3 \text { years }\end{array}$ & $\begin{array}{l}0 \\
1\end{array}$ \\
\hline S7 & Tumor characteristics (size and invasion) & $\begin{array}{l}\text { Adenoma size }<5 \mathrm{~mm} \\
\text { Adenoma size } \geqslant 5 \mathrm{~mm} \text { and/or CS invasion }\end{array}$ & $\begin{array}{l}0 \\
1\end{array}$ \\
\hline
\end{tabular}

24-h UFC, 24-h urine-free cortisol; BMI, body mass index; BP z score, blood pressure z score; CS, cavernous sinus; DBP, diastolic blood pressure; DM, diabetes mellitus; DM-Dx, diabetes mellitus diagnosis; DM-Tx, diabetes mellitus treatment with insulin or metformin at diagnosis; FPG, fasting plasma glucose; HTN-Tx, on medication for hypertension at diagnosis; IGT, impaired glucose tolerance; MSC, midnight serum cortisol; SBP, systolic blood pressure; $t$ (to diagnosis), duration from first symptoms to diagnosis.

SI conversion factors: to convert fasting plasma glucose to mmol/l, multiply values by 0.0555 . CD severity Score = sum of S1-S7.

diabetes are known to be the main determinants of cardiovascular events and mortality. We wanted to capture these signs in children as important determinants of overall disease severity (13-16). In the current study, we examined data to assess $\mathrm{CD}$ severity at presentation and risk for persistent disease and/or relapse.

\section{METHODS}

\section{Subjects}

Patients with $\mathrm{CD} \leqslant 18$ years at the time of transsphenoidal surgery were consecutively treated at the National Institutes of Health Clinical Center from 1 January 1997 to 1 January 2015. Informed consent and assent was obtained from all patients. The Institutional Review Boards of the Eunice Kennedy Shriver National Institute of Child Health and Human Development National Institutes of Health approved the research protocol (Clinical Trial Registration number: NCT00001595).

\section{Study Design}

Patients were admitted for pre-operative testing within 1 month of their surgical date to confirm CD following a standardized protocol. A venous sampling catheter was placed at least $2 \mathrm{~h}$ before the test; cortisol and adrenocorticotropic hormone levels were drawn at 2330 and 2400 hours; these values were averaged to become the 'average' late-night cortisol value. Pre-operative evaluation included (i) 24-h urinary-free cortisol, (ii) average late-night (2330 and 2400 hours) and morning (0730 and 0800 hours) serum cortisol levels, and (iii) average morning (0730 and 0800 hours) plasma adrenocorticotropic hormone levels. The initial visit data included age at surgery, gender, race/ethnicity (by self-report), number of transsphenoidal surgeries, years of hypercortisolism (as determined by a review of the growth chart and age of crossing percentiles for height and weight, as well as self-report of onset of physical symptoms of $\mathrm{CD}$ ), height, height $z$ score, weight, BMI, and BMI $z$ score. BMI $\left(\mathrm{kg} / \mathrm{m}^{2}\right)$ and BMI-for-age $z$ scores were calculated from the Centers for Disease Control and Prevention (CDC) growth charts (17). Surgical outcome, length of follow-up, as well as rates of recurrent CD were collected. A 10-point index for rating severity in pediatric CD (CD severity) (Table 1) was devised on thr basis of a review of the most clinically relevant manifestations in children, the co-morbidities associated with $\mathrm{CD}$, and the independent association of each factor with risk for a poor clinical outcome. Seven clinical features were selected on the basis of their frequency and importance. Degree of hypercortisolemia, impaired glucose tolerance, and hypertension were graded on an ordinal 3-point scale (0-2) with pre-defined cutoffs based on severity. Height and BMI $z$ scores, duration of disease, tumor size, and presence of tumor invasion were graded on an ordinal 2-point scale $(0-1)$. Total severity scores ranged from 0 to 10 . Estimated income data were obtained from US Census and World Bank databases, using zip codes of residence for US census data $(18,19)$. Prevalence of obesity in children by state was extrapolated from the Data Resource Center for Child and Adolescent Health, a project of the Child and Adolescent Health Measurement Initiative (CAHMI-2011) where obese children with BMI $z$ score $\geqslant 95 \%$ ile are reported by state (Table 2) (20). Access to pediatric endocrinologists by state was based on the relative distribution of American Board of Pediatrics-certified Pediatric Endocrinology Diplomates by state as of 12/31/2012 (Table 2) (21). Status of country in terms of whether or not it is developed was based on the International Monetary Fund's World Economic Outlook Report, April 2015 (22).

\section{Data Analysis}

Results were described as frequencies and percentages, or as means and SD's, unless otherwise indicated. Groups were defined on the basis of self-reported racial and ethnic descriptions, and were categorized as HI/AA if they indicated Hispanic or Latino ethnicity or indicated Black/African-American race regardless of ethnicity. The non-HW category consisted of those who indicated not being Hispanic or Latino for ethnicity and being white for race. Those not meeting these definitions were excluded. Data were assessed for their distributions and log-transformed as necessary. Continuous data were compared using the two-sample $t$-test or Wilcoxon rank-sum test as applicable; categorical data were compared by means of the $\chi^{2}$ or Fisher's exact test, and the Kruskal-Wallis test was used for singly ordered categories. Simple and multiple regression analyses were carried out to assess the relation between explanatory variables (race/ ethnicity, age, gender, prevalence of obesity in children by state, estimated median income per zip-code, access to pediatric 


\section{Articles | Gkourogianni et al.}

Table 2. Demographic characteristics of 129 children with Cushing disease, stratified by race/ethnicity

\begin{tabular}{|c|c|c|c|c|c|c|}
\hline Variable & Hispanic $(n=36)$ & $\begin{array}{c}\text { African-American } \\
(n=9)\end{array}$ & $\begin{array}{l}\text { Non-Hispanic White } \\
\qquad(n=84)\end{array}$ & $P$ value ${ }^{\mathrm{a}}$ & $\begin{array}{l}\text { Hispanic or African- } \\
\text { American }(n=45)\end{array}$ & $P$ value ${ }^{\mathrm{b}}$ \\
\hline Age (years) & $13.4 \pm 3.5$ & $13.8 \pm 2.2$ & $12.9 \pm 3.2$ & 0.61 & $13.5 \pm 3.3$ & 0.34 \\
\hline BMI z score & $1.9 \pm 0.8$ & $2.4 \pm 0.4$ & $2.1 \pm 0.7$ & 0.25 & $2.0 \pm 0.8$ & 0.64 \\
\hline Height z score & $-1.4 \pm 1.2$ & $-2.0 \pm 1.1$ & $-1.1 \pm 1.1$ & $0.030 *$ & $-1.6 \pm 1.2$ & $0.022^{*}$ \\
\hline Gender (F) & $21(58 \%)$ & $3(33 \%)$ & $40(48 \%)$ & 0.35 & $24(53 \%)$ & 0.58 \\
\hline Median income & $\$ 29,406 \pm \$ 36,034$ & $\$ 66,128 \pm \$ 34,935$ & $\$ 59,231 \pm \$ 31,314$ & $<0.001^{\mathrm{C} *}$ & $\$ 37,466 \pm \$ 38,564$ & $<0.001^{* *}$ \\
\hline $\begin{array}{l}\text { Prevalence of obesity } \\
\text { in resident state }\end{array}$ & & & & 0.63 & & 0.76 \\
\hline$\geqslant \geq 20.1 \%$ & 0 & $1(11.1 \%)$ & 9 (12.3\%) & & $1(4.6 \%)$ & \\
\hline $15.1-20 \%$ & $3(23.1 \%)$ & $4(44.4 \%)$ & $22(30.1 \%)$ & & $7(31.8 \%)$ & \\
\hline $10.1-15 \%$ & $10(76.9 \%)$ & $4(44.4 \%)$ & 37 (50.7\%) & & $14(63.6 \%)$ & \\
\hline $5.1-10 \%$ & 0 & 0 & $5(6.9 \%)$ & & 0 & \\
\hline $0-5 \%$ & 0 & 0 & 0 & & 0 & \\
\hline $\begin{array}{l}\text { Pediatric endocrinologist } \\
\text { per-capita score }\end{array}$ & $3.9 \pm 1.0$ & $3.6 \pm 1.5$ & $3.3 \pm 1.2$ & 0.29 & $3.7 \pm 1.2$ & 0.097 \\
\hline $\begin{array}{l}\text { Individuals from a } \\
\text { developing country }\end{array}$ & $18(50 \%)$ & 0 & $3(3.6 \%)$ & $<0.001^{f_{*}}$ & $18(40.0 \%)$ & $<0.001 * *$ \\
\hline
\end{tabular}

BMI, body mass index; F, female; US, United States.

Data are mean \pm SD or frequency (percent). Sums may not add up to total numbers due to being missing or not applicable (i.e., state information for non-US patients).

${ }^{*} P \leqslant 0.05,{ }^{* *} P \leqslant 0.01$.

a $P$ values: comparing all three groups

${ }^{b} P$ values: comparing combined Hispanic or African-American to non-Hispanic White

${ }^{c}$ Post hoc-corrected $P=0.011$ for Hispanic vs. African-American and $P<0.001$ for Hispanic vs. non-Hispanic White.

dercentage of children with BMI $z$ score at $\geqslant 95 \%$ ile for each patient's resident US state, scores of 1-5 correspond as follows: 1 ( $\geqslant 20.1 \%), 2$ (15.1-20\%), 3 (10.1-15\%), 4 (5.1$10 \%)$, and $5(0-5 \%)$.

ePediatric endocrinologists per-capita score for each patient's resident US state: 0 (no certified specialists), 1 (1:150,000+), 2 (1:100,000-149,000), 3 (1:75,000-99,999), 4 (1:50,00074,999), and $5(1: 1-49,999)$

${ }^{f}$ Post hoc-corrected $P=0.013$ for Hispanic vs. African-American and $P<0.001$ for Hispanic vs. non-Hispanic White.

endocrinologists, and whether an individual resided in a developing country) and disease severity outcome as scored by the index described above. The Kaplan-Meier method was used to compute the survivor function for time to recurrence that were compared between the two groups using the log-rank test. Data were considered statistically significant if the resulting $P$ value was $<0.05$, or the relative risk $95 \%$ confidence interval excluded 1.0. Corrected $P$ values are reported for post hoc comparisons, which utilized the Bonferroni method. Analyses were carried out using SAS v9.4 (SAS Institute, Cary, NC).

\section{RESULTS}

From an initial population of 139 pediatric CD patients admitted to the National Institutes of Health Clinical Center between the years 1997 and 2015, data were collected retrospectively from records of children diagnosed with $\mathrm{CD}$ at age $\leqslant 18$ years (64 girls and 65 boys; median age 13.4 years). The year 1997 coincided with the initiation of our protocol entitled 'A Clinical and Genetic Investigation of Pituitary Tumors and Related Hypothalamic Disorders', in which these patients were enrolled, as well as the year at which standardization of racial and ethnic designations was revised by the Office of Management and Budget (23). Patient racial/ethnic groups were distributed as $34.9 \%(n=45) \mathrm{HI} / \mathrm{AA}$ and $65.1 \%(n=84)$ non-HW. Asian patients $(n=6)$ and those with unknown race/ethnicity $(n=4)$ were excluded from the study; thus, a total of 129 patients were included in the analysis. The complete data sets, stratified by race/ethnicity showing HI, AA, and non-HW data individually as well as HI and AA grouped together, are presented in Tables 2 and 3.

The median income was higher in the non-HW group, and fewer participants from the non-HW group were from developing countries (both $P<0.001$; Table 2) compared with those in the HI/AA group. The developing countries where our patients reside, followed by the number of patients from each country, are as follows: Peru (5), Chile (4), and Brazil (3), and one child each from Colombia, Ecuador, Guatemala, Venezuela, Uruguay, and Argentina.

Midnight cortisol levels were higher in the HI/AA group $(23.3 \pm 22.0$ vs. $16.2 \pm 8.5 \mu \mathrm{g} / \mathrm{dl}, P=0.040)$, and height SD score was more severely affected $(-1.6 \pm 1.2$ vs. $-1.1 \pm 1.1$, $P=0.022)$, when compard with the non-HW group. Tumor size was also larger in the HI/AA group $(6.3 \pm 7.1$ vs. $3.2 \pm 3.3 \mathrm{~mm}, P=0.007)$.

The mean CD severity score of the HI/AA group was worse than that of the non-HW group $(4.9 \pm 2.0$ vs. $4.1 \pm 1.9$, $P=0.023$ ). Simple regression analysis of disease severity revealed that age was an independent explanatory variable of disease severity as measured by $\mathrm{CD}$ severity $\left(r_{\mathrm{p}}=0.23\right.$, $P=0.008$ ), whereas gender, number of pediatric endocrinologists per capita, median income, prevalence of obesity in the patient's state of residence, and whether or not an individual resides in a developing country were not independent driving factors. Considering these effects together in multiple 
Table 3. Patient characteristics of 129 children with Cushing disease as they relate to severity of presentation, stratified by race/ethnicity

\begin{tabular}{|c|c|c|c|c|c|c|}
\hline Variable & Hispanic $(n=36)$ & $\begin{array}{l}\text { African-American } \\
\qquad(n=9)\end{array}$ & $\begin{array}{l}\text { Non-Hispanic White } \\
\quad(n=84)\end{array}$ & $P$ value $^{\mathrm{a}}$ & $\begin{array}{l}\text { Hispanic or African-American } \\
\qquad(n=45)\end{array}$ & $P$ value ${ }^{\mathrm{b}}$ \\
\hline MSC ( $\mu \mathrm{g} / \mathrm{dl})$ & $25.1 \pm 23.9$ & $16.1 \pm 10.3$ & $16.2 \pm 8.5$ & $0.038^{C_{*}}$ & $23.3 \pm 22.0$ & $0.040^{*}$ \\
\hline 24-h UFC ( $\mu \mathrm{g} / 24 \mathrm{~h})$ & $905.3 \pm 2,561.1$ & $453.5 \pm 612.2$ & $263.4 \pm 237.7$ & 0.21 & $815.0 \pm 2,306.3$ & 0.13 \\
\hline Adenoma size (mm) & $6.0 \pm 6.7$ & $7.4 \pm 8.9$ & $3.2 \pm 3.3$ & $0.025^{d_{*}}$ & $6.3 \pm 7.1$ & $0.007^{* *}$ \\
\hline CS invasion & $4(11.1 \%)$ & $1(11.1 \%)$ & $3(3.6 \%)$ & 0.22 & $5(11.1 \%)$ & 0.13 \\
\hline DM-Tx & $6(16.7 \%)$ & $1(11.1 \%)$ & $4(4.8 \%)$ & 0.087 & $7(15.6 \%)$ & $0.049 *$ \\
\hline HTN-Tx & $9(25.0 \%)$ & $3(33.3 \%)$ & $12(14.3 \%)$ & 0.17 & $12(26.7 \%)$ & 0.10 \\
\hline$t$ (to diagnosis) (years) & $2.8 \pm 2.1$ & $4.2 \pm 2.3$ & $2.5 \pm 1.5$ & 0.089 & $3.1 \pm 2.2$ & 0.37 \\
\hline $\begin{array}{l}C D \text { severity score }(0- \\
10)\end{array}$ & $4.6 \pm 1.8$ & $6.0 \pm 2.5$ & $4.1 \pm 1.9$ & $0.012^{\mathrm{e}_{*}}$ & $4.9 \pm 2.0$ & $0.023^{*}$ \\
\hline \multicolumn{7}{|c|}{ 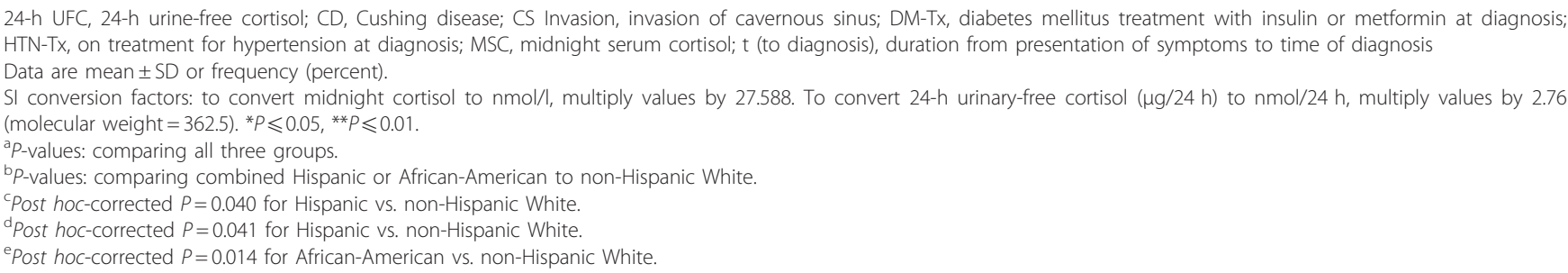 } \\
\hline
\end{tabular}

regression models confirmed that race $(P=0.027)$ and older age $(P=0.014)$ were the most important predictors of worse CD severity.

\section{Follow-Up}

Of the 129 children in this study, 124 achieved remission after surgery. Of the five patients who did not achieve remission after surgery, two underwent radiation therapy and three were managed with ketoconazole. Long-term follow-up was available in 112 patients (87\%) with mean postoperative follow-up of $45.5 \pm 39.9$ months, median follow-up of 27.9 months (interquartile $\mathrm{ra}=13.2-68.7$ months), and range of 1.4-158.7 months. Interestingly, a follow-up was available in more non-HW individuals, 78/84 (93\%), as compared with HI/AA individuals, $34 / 45(76 \%)(P=0.012)$, and mean CD severity was significantly worse in those individuals without long-term follow-up $(5.4 \pm 1.7$ vs. $4.2 \pm 2.0, \quad P=0.017)$. Thirteen of the 108 patients with a long-term follow-up (excluding those who had persistent disease) developed recurrent $\mathrm{CD}$ over the course of the study, with a mean time to recurrence of $49.2 \pm 28.2$ months (range of 1.9-111.3 months). Of these individuals, one underwent bilateral adrenalectomy, three were treated with pituitary radiation therapy, two underwent repeat pituitary surgery in addition to pituitary radiation therapy, six underwent repeat transsphenoidal surgery alone, and one individual is awaiting repeat surgery. Two individuals are currently deceased-one secondary to suicide and another from pulmonary embolism. Overall, 13 patients had recurrence of disease after surgery and five had persistent disease after surgery. As expected, CD severity was worse among those individuals with recurrent disease $(5.1 \pm 1.7$ vs. $4.1 \pm 1.9, P=0.041)$. Among those individuals with a long-term follow-up or known persistence of disease after surgery, a higher proportion of individuals in

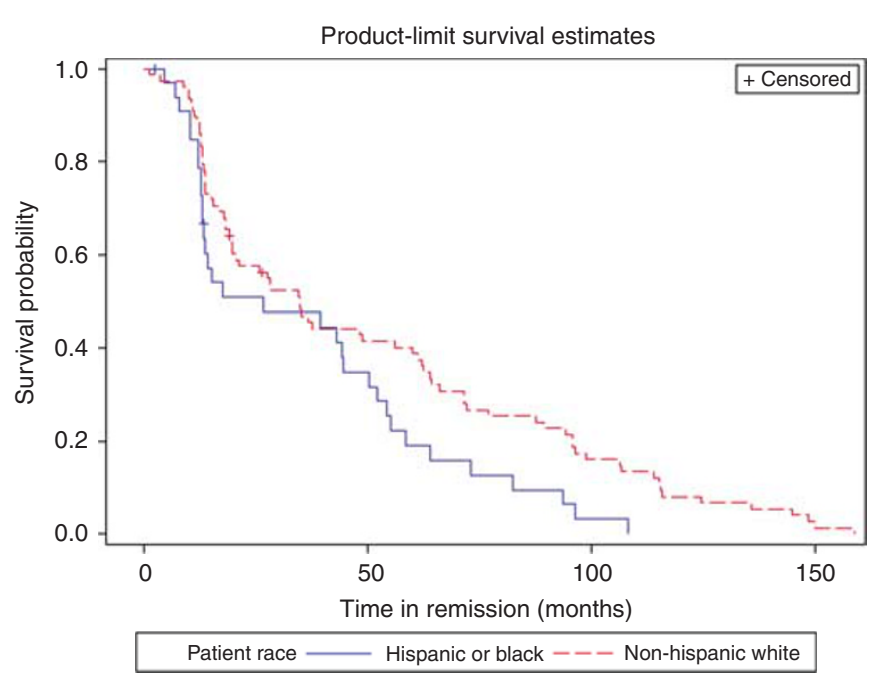

Figure 1. The Kaplan-Meier curves for disease-free survival in HI/AA and non-HW study groups (time in remission): HI/AA median $=26.8$ (95\% Cl: 13.2-50.3) months vs. non-HW median $=34.8$ (95\% Cl: $19.7-$ 60.1) months; log-rank $P=0.028$ ).

the HI/AA group had a lack of initial cure or recurrence (10 out of $35 \mathrm{AA} / \mathrm{HI}$ individuals, 28.6\%) when compared with the non-HW group ( 8 out of $78,10.3 \%, P=0.024$ ). The relative risk for persistent $\mathrm{CD}$ combined with recurrence was 2.8 times higher in the HI/AA group as compared with that in the non-HW group (95\% confidence interval: 1.2-6.5). When disease-free survival was compared (time in remission), the median for HI/AA individuals was 26.8 (95\% confidence interval: 13.2-50.3) months, whereas that for non-HW individuals was 34.8 (95\% confidence interval: 19.7-60.1) months; log-rank $P=0.028$ (Figure 1). 


\section{Articles | Gkourogianni et al.}

\section{DISCUSSION}

Our findings show disproportionately higher severity of $\mathrm{CD}$ in HI/AA patients both preoperatively and postoperatively. SES was also lower in the HI/AA individuals. SES status contributes to health disparities as poverty, lack of health insurance, and poor access to care cause the medically underserved to bear a greater burden of disease than the general population. In the United States, both AfricanAmerican and Hispanic/Latino minority groups have lower SES, decreased access to healthcare, and worse outcomes across a number of health indicators compared with Whites (24). For all cancer sites combined, residents of poorer countries have higher death rates from cancer compared with those residing in more affluent countries (25). Later stage at diagnosis of cancer has been associated with lower SES (6). It was observed that the HI/AA children in our study had a higher rate of diagnosis at an advanced stage; children of lowincome families may have limited access to specialized care, and cancer diagnosis, including CD, may be delayed $(26,27)$.

One potential cause for worse CD severity scores in the HI/AA group may be the higher prevalence of obesity in minority children, which may lead practitioners to overlook one of the presenting symptoms of $\mathrm{CD}$. The prevalence of obesity in non-Hispanic White youth and young adults is $14.1 \%$, whereas the prevalence in Hispanic/Latinos and African-Americans is $22.4 \%$ and $20.2 \%$, respectively (28). In addition, delayed referral for surgical intervention or participation in a clinical trial for a rare disease may be contributing factors for the higher severity in HI/AA children with CD. Transsphenoidal surgery is the treatment of choice, with optimal outcomes when performed at tertiary referral centers (1). Racial and socioeconomic disparities have been found specifically for children in terms of access to highvolume neuro-oncological care (29). In addition, minorities with cancer are under-represented in pediatric oncology research protocols (30).

Social factors including race/ethnicity, educational level, language/culture barriers, income, poverty, unemployment, and lack of health insurance all contribute to health outcomes, in addition to one's genetic background $(10,25)$. Progress in pediatric cancer survival rates is highly attributable to early detection, advances in therapeutic protocols, and patients' participation in clinical trials (7). The association of younger age, smaller tumor size, and absence of cavernous sinus invasion (experienced surgeon and center of referral) with lasting biochemical remission suggests that earlier diagnosis when the tumor is small and noninvasive will enhance a longterm outcome; late pituitary $\mathrm{CD}$ diagnoses are associated with more co-morbidities and higher recurrence and mortality rates (3). Awareness of the signs and symptoms of $C D$ needs to be increased among care providers. We suggest that the American Cancer Society and American Cancer Society Cancer Action Network together should influence public policies and promote patients' navigators and medical homes to reduce health disparities (31-33).
Our study is the first to introduce a combined severity score for childhood CD, which we observed to be associated with outcome; that is, the higher the severity score, the higher the likelihood of recurrence and failure to cure. One limitation of our study associated with the use of a novel severity score for pediatric $\mathrm{CD}$ is that it has yet to be validated in a larger cohort. Given the rarity of childhood CD, we will work toward collaborating with other institutions to validate the performance of the severity scoring system. Another limitation of our study is the use of residential zip codes as a proxy for income that may not account for within-area variation. In addition, patient health insurance status is not collected at the National Institutes of Health Clinical Center, as patients are participating in a research study.

HI/AA children had comparatively more severe disease presentation of CD compared with non-HW children, and a nearly threefold higher risk for persistent $\mathrm{CD}$ or recurrent disease after surgery. Our data show that the driving forces for the discrepancy in disease severity are older age at the time of treatment as well as race. Delayed diagnosis and treatment and lack of access to care for these underserved children may contribute to presentation at a later age and to increased morbidity. We speculate that delayed diagnosis and treatment, barriers to access to medical care, and poorer-quality healthcare for these underserved patients may contribute to presentation at a later age and increased morbidity. Additional research is needed to identify potential modifiable factors that may improve care for these patients.

\section{STATEMENT OF FINANCIAL SUPPORT}

This research was supported in part by the Intramural Research Program of the Eunice Kennedy Shriver National Institute of Child Health and Human Development, National Institutes of Health.

Disclosure: The authors declare no conflict of interest.

\section{REFERENCES}

1. Stratakis CA. Cushing syndrome in pediatrics. Endocrinol Metab Clin North Am 2012;41:793-803.

2. Lodish M. Cushing's syndrome in childhood: update on genetics, treatment, and outcomes. Curr Opin Endocrinol Diabetes Obes 2015;22: $48-54$.

3. Lonser RR, Wind JJ, Nieman LK, Weil RJ, DeVroom HL, Oldfield EH. Outcome of surgical treatment of 200 children with Cushing's disease. J Clin Endocrinol Metab 2013;98:892-901.

4. Siegel R, Ward E, Brawley O, Jemal A. Cancer statistics, 2011: the impact of eliminating socioeconomic and racial disparities on premature cancer deaths. CA Cancer J Clin 2011;61:212-36.

5. Linabery AM, Ross JA. Childhood and adolescent cancer survival in the US by race and ethnicity for the diagnostic period 1975-1999. Cancer 2008;113:2575-96.

6. Clegg LX, Reichman ME, Miller BA, et al. Impact of socioeconomic status on cancer incidence and stage at diagnosis: selected findings from the surveillance, epidemiology, and end results: National Longitudinal Mortality Study. Cancer Causes Control 2009;20:417-35.

7. Zeng C, Wen W, Morgans AK, Pao W, Shu XO, Zheng W. Disparities by race, age, and sex in the improvement of survival for major cancers: results from the National Cancer Institute Surveillance, Epidemiology, and End Results (SEER) Program in the United States, 1990 to 2010. JAMA Oncol 2015;1:88-96. 
8. Kadan-Lottick NS, Ness KK, Bhatia S, Gurney JG. Survival variability by race and ethnicity in childhood acute lymphoblastic leukemia. JAMA 2003;290:2008-14.

9. Petridou ET, Sergentanis TN, Perlepe C, et al. Socioeconomic disparities in survival from childhood leukemia in the United States and globally: a meta-analysis. Ann Oncol 2015;26:589-97.

10. Bhatia S. Disparities in cancer outcomes: lessons learned from children with cancer. Pediatr Blood Cancer 2011;56:994-1002.

11. Lodish MB, Sinaii N, Patronas N, et al. Blood pressure in pediatric patients with Cushing syndrome. J Clin Endocrinol Metab 2009;94:2002-8.

12. Aicardi G, Benso L, Vignolo M, et al. Dose-dependent effects of deflazacort and prednisone on growth and skeletal maturation. $\mathrm{Br} \mathrm{J}$ Rheumatol 1993;32 (Suppl 2): 39-43.

13. Etxabe J, Vazquez JA. Morbidity and mortality in Cushing's disease: an epidemiological approach. Clin Endocrinol (Oxf) 1994;40:479-84.

14. Bolland MJ, Holdaway IM, Berkeley JE, et al. Mortality and morbidity in Cushing's syndrome in New Zealand. Clin Endocrinol 2011;75:436-2.

15. Clayton RN, Raskauskiene D, Reulen RC, Jones PW. Mortality and morbidity in Cushing's disease over 50 years in Stoke-on-Trent, UK: audit and meta-analysis of literatureJ Clin Endocrinol Metab 2011;96:632-42.

16. Lambert JK, Goldberg L, Fayngold S, Kostadinov J, Post KD, Geer EB. Predictors of mortality and long-term outcomes in treated Cushing's disease: a study of 346 patients. J Clin Endocrinol Metab 2013;98:1022-30.

17. Kuczmarski RJ, Ogden CL, Grummer-Strawn LM, et al. CDC growth charts: United States. Adv Data 2000;314: 1-27.

18. The World Bank Gross national income per capita ranking table based on the World Bank Atlas method and purchasing power parity 2015. (http://data. worldbank.org/data-catalog/GNI-per-capita-Atlas-and-PPP-table).

19. The United States Census 2012 Economic Census Data. (https://www. census.gov/econ/geo-zip.).

20. Data Resource Center for Child and Adolescent Health National Survey of Children's Health (NSCH) Child and Adolescent Health Measurement Initiative 2011. (http://www.childhealthdata.org.).

21. American Board of Pediatrics. Pediatric workforce data, Number of ABP Pediatric Endocrinology Diplomates by State 2015. (https://www.abp.org/ content/workforce-data).
22. International Monetary Fund Fund. World Economic Outlook Database 2015. (http://www.imf.org/external/pubs/ft/weo/2015/01/weodata/index. aspx).

23. OMB (Office of Management and Budget). Revisions to the standards for the classification of federal data on race and ethnicity. Fed Regist 1997;62: 58781-90.

24. Halpern MT, Ward EM, Pavluck AL, Schrag NM, Bian J, Chen AY. Association of insurance status and ethnicity with cancer stage at diagnosis for 12 cancer sites: a retrospective analysis. Lancet Oncol 2008;9:222-31.

25. Ward E, Jemal A, Cokkinides V, et al. Cancer disparities by race/ethnicity and socioeconomic status. CA Cancer J Clin 2004;54:78-93.

26. Mukherjee D, Zaidi HA, Kosztowski T, et al. Disparities in access to neuro-oncologic care in the United States. Arch Surg 2010;145:247-53.

27. Mukherjee D, Zaidi HA, Kosztowski T, et al. Predictors of access to pituitary tumor resection in the United States, 1988-2005. Eur J Endocrinol 2009;161:259-65.

28. Ogden CL, Carroll MD, Lawman HG, et al. Trends in obesity prevalence among children and adolescents in the United States, 1988-1994 through 2013-2014. JAMA 2016;315:2292-9.

29. Mukherjee D, Kosztowski T, Zaidi HA, et al. Disparities in access to pediatric neurooncological surgery in the United States. Pediatrics 2009;124:e688-96.

30. Aristizabal P, Singer J, Cooper R, et al. Participation in pediatric oncology research protocols: Racial/ethnic, language and age-based disparities. Pediatr Blood Cancer 2015;62:1337-44.

31. Moy B, Polite BN, Halpern MT, et al. American Society of Clinical Oncology policy statement: opportunities in the patient protection and affordable care act to reduce cancer care disparities. J Clin Oncol 2011;29: 3816-24.

32. Srinivasan S, Williams SD. Transitioning from health disparities to a health equity research agenda: the time is now. Public Health Rep 2014;129 (Suppl 2): 71-6.

33. Strickland BB, Jones JR, Ghandour RM, Kogan MD, Newacheck PW. The medical home: health care access and impact for children and youth in the United States. Pediatrics 2011;127:604-11. 